\title{
Research to action: Nurse practitioners in the emergency department, Emergency Department Transition Clinic and Intravenous Therapy Clinic at Strathcona Community Hospital
}

\author{
Wesley Shand, Daris Klemmer, Shereen Grubb, Susan Chesney, Ben Olsen, Lawrence So
}

\section{Abstract}

This article is about nurse practitioners effectiveness working in the Strathcona emergency department (ED), as well as the efficacy of two nurse practitioner-led clinics that run parallel to the ED. Prior to opening Strathcona Community Hospital in 2014, site leadership were tasked with developing an innovative care model with the aim of improved patient safety and quality of care delivered, incorporating a nurse practitioner (NP) model. There are NPs in three areas at Strathcona Community Hospital. NPs work directly in the ED, assessing and treating patients autonomously and with emergency physician collaboration. They also complete diagnostic and microbiology reviews and perform triage liaison nurse practitioner duties. There is also an NP led Emergency Department Transition Clinic for urgent or emergent follow up patients from the ED. Lastly, the NP-led Intravenous Therapy Clinic was developed to see patients previously attended through the ED for IV antibiotic and other IV non-antibiotic treatments, while supporting increased community access to IV treatments. Evaluation of the three areas was completed using qualitative and quantitative methods over the period of 2015-2018. Statistical analysis was completed by the Alberta Health Services workforce team. Outcomes included reduced patient return visits, decreased wait times and patients leaving without treatment, and high patient satisfaction. Other results included improved staff satisfaction, facilitation of continuity of care and avoiding unnecessary ED visits.

\section{Introduction}

$\mathrm{N}$ urse practitioners (NPs) need to be more fully integrated into the healthcare system to ensure the sustainability and quality of healthcare expected by today's population. Current health system constraints result in inconsistent primary healthcare services and suboptimal health teams
(El-Jardali \& Lavis, 2011). Nurse practitioners are able to contribute to the health system from primary care to acute care including emergency departments (ED). With gaps in healthcare services, some patients will present to ED for emergent and non-urgent reasons. According to Jennings, Clifford, Fox, \& O'Connell (2015), “The delivery of quality care in the ED is emerging as one of the most important service indicators in health delivery." NPs are well suited to care for patients in ED and ambulatory care settings. This article describes the integration of NPs into a suburban hospital with a stand-alone emergency department and two separate ambulatory care settings. Intravenous Therapy Clinic (IVT) is an outpatient clinic providing 16 hour/day IV therapy services and Ambulatory Care Clinic (ACC) where there is a NP-run Emergency Department Transition Clinic (EDTC) that runs five days per week to provide follow-up appointments for patients recently seen in the ED. The three areas will be discussed separately under three headings: ED/IVT/EDTC.

\section{Demographics of Sherwood Park/Strathcona County}

Strathcona Community Hospital ( $\mathrm{SCH}$ ) is a hospital that opened in 2014 to meet an increased health need in Sherwood Park, Strathcona County, and surrounding areas (Tessera \& Gibbons-Reid, 2015). It has a variety of services including: $\mathrm{ED}$, laboratory, diagnostic imaging (including a computerized tomography (CT) scanner); Ambulatory Care Clinics (ACC); and mental health. Sherwood Park has a population of 83,555 people (2016); Strathcona County has a population of 18,232 (2016) for a total population of 101,787 served by SCH (Government of Alberta, 2017). The geographical area served is located approximately $30 \mathrm{~km}$ outside the urban area of Edmonton, Alberta; population 932,546 (Canada, 2016). Sherwood Park and Strathcona Country are affluent communities compared to the rest of Alberta with $43 \%$ having an income of greater than $\$ 100,000$ after tax compared to the rest of Alberta at 27\% (Government of Alberta, 2017). Sherwood Park residents have an above average completion of high school and post-secondary education. It has a similar population of First Nations/ Indigenous compared to the rest of Alberta (Government of Alberta, 2017). 


\section{Strathcona Community Hospital}

Prior to opening $\mathrm{SCH}$, site leadership were tasked with developing an innovative care model: "do it different" with the aim of improved patient safety and quality of care delivered. The three pillars to $\mathrm{SCH}$ are: 1. Collaboration; 2. Multidisciplinary; and 3. Integration. The NP model of care was incorporated into the site integrating the pillars with an innovative model of care that has been implemented in ED, Intravenous Therapy Clinic (IVT) and ACC settings. Having a NP in all three services is integral at a hospital site that is a stand-alone ED with no in-patient beds.

Nurse practitioners are Masters- and/or PhD-level registered nurses with additional educational preparation and clinical experience. They possess and demonstrate the competencies to autonomously diagnose, order and interpret diagnostic tests, prescribe pharmaceuticals and perform specific procedures within their legislated scope of practice (Canadian Nurses Association, 2006). Nurse practitioners practising in Alberta have a broad autonomous and independent scope of practice and are accountable and responsible for their own practice (CARNA, 2017). To ensure NPs could be fully integrated in all areas, SCH NPs are rostered/trained in Family All Ages and are able to care for patients across the spectrum from birth to death.

Involving NPs in all care areas at $\mathrm{SCH}$, allows for acute and emergent issues to be assessed, and the transition and follow-up back to primary care completed. Nurse practitioners can act as the touch point throughout the patient's healthcare experience, allowing appropriate community services and referrals completed as necessary.

\section{NP Service areas}

Emergency Department: Nurse practitioners work directly in the ED, assessing and treating patients triaged as Canadian Triage Acuity Scale (CTAS) 4/5 autonomously and with emergency physician collaboration as needed and for CTAS $2 / 3$. They also complete the diagnostic and microbiology review and perform triage liaison provider (TLP) duties. The TLP duties involve engaging patients in the waiting room and initiating diagnostics and symptomatic treatments to promote comfort, decrease wait times in the ED, and decrease the number of patients who leave without treatment (Nestler, et al., 2012).

Intravenous Therapy Clinic (IVT): The NP-led IVT was developed to manage patients previously seen through the ED, for intravenous antibiotic and non-antibiotic infusions. The NPs in IVT clinic also have a collaborative partnership with Infectious Disease (ID)/Internal Medicine. In collaboration with the medical director and the ID physician clinical practice guidelines were developed for presentations that can be managed autonomously by the NP, as well as patients that would initially be followed by the NP while waiting for ID consultant review. The IVT NP also reviews/triages referrals from zone specialists and community General practitioners (GPs) for both antibiotic and non-antibiotic patients. The NP completes initial history and physical exam on all patients accepted into the clinic, plus appropriate scheduled reassessments on patients managed autonomously by the NP. The NP also manages acute/urgent concerns with any IVT patient, for example pain management, antibiotic changes based on culture sensitivities, and nausea management. In other sites, IV services are often provided via the ED/in-patient units. This model provides high-quality medical care, while keeping patients out of hospital and at home. It also allows for patients to be treated outside of the emergency department, thus freeing up time/resources/providers in the ED and likely decreasing overall costs associated with the ED visit.

\section{Ambulatory Care Clinic (ACC):}

The ACC provides outpatient services for a variety of subspecialty consulting services. The EDTC is an innovative NP-led clinic open five days per week. The EDTC provides timely, high-quality care for patients seen in the emergency department that require ongoing and transitional care. This ensures patients do not get lost during follow-up. Patients who are unable to get timely GP follow-up or don't have a GP can be referred to this clinic. Examples of patients seen in this clinic are those needing diagnostic imaging follow-up; repeat laboratory tests; and those who require reassessment of common ED presentations including chronic obstructive pulmonary disease, asthma, bronchitis, dehydration, abdominal pain, and acute wound care (burns, complex lacerations, and debridement).

\section{Methods}

The evaluation of the nurse practitioner model at the Strathcona Community Hospital has been ongoing annually beginning in 2014. All evaluations have been assisted by the Alberta Health Services (AHS) evaluation team or more recently AHS Clinical Workforce Planning within the AHS Health Professions Strategy and Practice team. The first year evaluation was completed using quantitative methods. Years two and three were quantitative and qualitative data. Year four utilized quantitative methods with added outcomes measures, workforce metrics and cost analysis.

Statistical analysis was completed by AHS Clinical Workforce Team. Statistical data was extracted from multiple sources including: Emergency Department Information System (EDIS), physician billing, National Ambulatory Care Reporting System (NACRS), Tableau [Seattle, Washington, United States of America], and e-CLINICIAN [Calgary, Alberta, Canada]. Year one, patient interviews were completed on patient satisfaction and the overall care received in ED from either the NP or emergency physician. Year two and three, the mixed method approach continued and focused on service delivery utilizing patient and caregiver surveys, staff and physician surveys, and focus group discussion with SCH NPs. Year four utilized a comparative approach to determine how $\mathrm{SCH}$ compared to other EDs and Urgent Care Centers throughout Alberta.

Ethical considerations were incorporated throughout the annual evaluations. No specific patient data was utilized but ethical considerations were considered for the survey questions and focus group. Specific to the patient satisfaction surveys, the survey tools were reviewed by a Second Opinion Reviewer as part of the Alberta Research Ethics Community Consensus Initiative (ARECCI) process [http://www.aihealthsolutions. $\mathrm{ca} /$ arecci/screening]. 


\section{Results}

\section{Emergency Department (ED)}

Year one data showed that NPs improve access to care and efficiency at SCH. It was also shown that NPs improve patient healthcare outcomes directly in the ED by providing direct patient care, reducing the number of patients who left without treatment (LWOT) and by reviewing DI and microbiology results. NPs provide care that is satisfying to patients; there was no statistical difference in patient satisfaction rates between NPs and emergency physicians (Tessera \& Gibbons-Reid, 2015).

Year two evaluation data also proved NPs improve access to care and improve patient flow in the ED. Surveys were completed by patients, caregivers, staff and physicians. Positive feedback from the patient/caregiver survey indicated that NPs treatment was prompt and that the patients felt treated with respect and with the highest level of professional expertise (Tessera \& GibbonsReid, 2016). The staff and physician survey had positive comments, such as NPs provide collaborative service, reduce ED wait times and can spend more time with the patients to provide additional supports/explanations.

Year 4 evaluation statistics/data include daily follow-up of all ED providers' results by the Emergency Pharmacist (EP) and NP for microbiology results; as well as, by the NP for diagnostic imaging results if radiology reporting was not completed prior to discharge (Olsen, Yu, \& So, 2018). In 2018-2019, 5,395 lab reports were reviewed and 751 follow-up calls were completed to patients with positive lab results. Examples of results requiring a telephone call included Group A streptococcus-positive throat swabs not empirically treated and urine cultures for urinary tract infection where the empiric therapy is resistant to the organism. In 2018-2019, 7,143 diagnostic imaging reports were reviewed and 287 calls to patients were completed. Patients would be called to be made aware of missed fractures on x-ray or CT scan. Appropriate referral/reassessments/consultation would be completed by the NP. These secondary consults and clinical supports highlight some of the invisible work that the NPs are providing to support the patients through the emergency department (Lutze, Fry, O’Connell, \& Coates, 2018).

As SCH is a relatively new ED, there were many LWOT patients when the hospital first opened. Nurse practitioners in the ED are tasked with following up on patients who leave before being seen by a provider or prior to completing treatment. Some patients that leave the ED are at risk due to delays in receiving medical care or for risk of decompensating at home (Rowe, et al., 2006). Communications with LWOT patients allows the opportunity for the NP to review the presenting complaint as well as current patient status, thus allowing for the most appropriate follow-up plan to be suggested. There were 1,748 of these calls made in 2018-2019.

The year-four NP evaluation also included revisits to the emergency department. Revisits were defined as the proportion of return visits to the ED within 72 hours. The revisit rate was one of the lowest amongst comparator sites in the Edmonton Zone (Olsen, Yu, \& So, 2018). This outcome has also been noted by (Lutze et al., 2018) also showed that emergency nurse practitioners had lower revisit rates. The year 4 data also showed the lowest time to physician for CTAS $2 \& 3$ and some of the lowest wait times in the Edmonton zone. The collaborative/integrative model at $\mathrm{SCH}$ has shown reduced patient return visits, decreased wait times and patients who LWOT. Other results in the NP evaluation data include improved staff satisfaction, facilitation of continuity of care and avoiding unnecessary ED visits with high patient satisfaction.

\section{Intravenous Therapy Clinic (IVT)}

The Strathcona Community Hospital IV Clinic was opened in Aug 2015. During the first year (June 2015-May 2016) 894 patients were seen (Tessera \& Gibbons-Reid, 2016). The number of patient's seen/unique visits/clinic capacities has steadily grown in the past four years. In the most recent evaluation year, 2017-2018, 1,162 patients were assessed/treated by nurse practitioners (Olsen et al., 2018). These patients include antibiotic related diagnoses and non-antibiotic medical therapies. Most patients are referred to IVT from the emergency department such as cellulitis, dental abscess, pneumonia, and pyelonephritis patients. Other patients include IV iron sucrose, intravenous immunoglobulin, blood transfusion, phlebotomy for hemochromatosis patients from the community. Three hundred and twenty consultations to other providers were completed, the majority to infectious diseases specialty to ensure optimal patient care was delivered. Daily assessments are completed for unwell patients who might otherwise be admitted to hospital (such as: pneumonia, pyelonephritis, or peritonsillar abscess). According to Claeys et al., a decreased rate of ED revisits/hospitalization supports the use of outpatient IV clinics for low acuity acute bacterial superficial skin infections requiring initial IV therapy (2015).

\section{Ambulatory Care Clinic (ACC)}

Year one evaluation data was positive and concluded utilization of the EDTC, by providing follow up of emergency patients, ensured that patients were not lost in the vast healthcare system (Tessera \& Gibbons-Reid, 2015). The SCH EDTC was opened when the SCH ED was opened in 2014. Since inception on opening of SCH EDTC, the clinic has increased in numbers: Year one; 1,114; Year two: 1,372; Year three: 1,484; Year four: 1,787; and year five: $2,300+$ with a subsequent $250+$ patients referred to this clinic but booked and seen as out patients through other clinics. The majority of these patients were referred from the ED, with a small amount being sent from the IVT for reassessments post-completion of IV therapy. Recently added to the EDTC NP duties is the review of ultrasounds ordered in the ED, to be completed urgently as an outpatient investigation. Between March 2018 and March 31, 2019, 685 patients were seen for ultrasound results and reassessments. Diverting these patients from the ED builds capacity in the ED. Dr. Tubman (SCH ED chief) noted that since this process has begun the overall numbers in ED have decreased (2019). Presentations to EDTC have had an impact on overall ED numbers and has helped to build capacity in the ED.

\section{Patient Experience}

Overall there is high satisfaction among patients receiving care at SCH ED and the supporting clinics-IVT and ACC. Year one evaluation data included $95 \%$ of patient satisfaction with the care in ACC. In the ED, there was no statistical significant 
difference between emergency physicians and NPs in patient satisfaction (Tessera \& Gibbons-Reid, 2015). Year two evaluation data included patient comments that they felt like the NP treated them with courtesy and respect. They also felt that medical information was explained in a way that they could understand. Eighty-nine percent of patients surveyed in the ED were satisfied with the quality of care they received by NPs in ED (Tessera \& Gibbons-Reid, 2016).

\section{Quality, Safety and Staff Feedback}

Taking into consideration the AHS six quality indicators: accessibility, acceptability, appropriateness, effectiveness, efficiency, and safety, our site has performed exceptionally well. The year three NP evaluation included a questionnaire to site staff, including physicians, regarding the NPs improving access to health services for $\mathrm{SCH}$ patients. Results included $92 \%$ of staff and physicians believing that NPs add value to $\mathrm{SCH}, 90 \%$ believed that NPs improve access to services and $82 \%$ believe that NPs improve patient flow in the emergency department (Tessera \& Gibbons-Reid, 2017).

\section{Discussion}

The three different areas that NPs work in the Strathcona Community Hospital prove that NPs can be integrated into a hospital. Emergency department NPs provide direct patient care, triage liaison practitioner duties, complete diagnostic imaging and microbiology review and follow-up. This ensures that no patients are lost to follow-up and ensures that high-quality safe care is delivered. The IV outpatient therapy clinic directly offloads patients from ED. Daily assessments of the higher acuity patients in the IV clinic provides for decreased hospital admissions. The patients are closely monitored for follow-up and consultation as required to ensure patient safety. The IV clinic also ensures appropriate resource allocation and medical collaboration. The EDTC clinic has many valuable attributes for improving the patient experience. They ensure urgent referral follow-up in an appropriate time. The NPs connect to specialists and complete referrals as necessary. The ultrasound follow-up builds extra capacity for ED. The clinic as a whole strives to ensure that no patient is lost in follow-up, while delivering quality patient care and enhancing patient satisfaction.

\section{Learnings}

There have been many learnings over the four-year-long course of evaluation. Recommendations were discussed for NP role implementation in different settings, i.e., hospitals. Nurse practitioner focus-group discussions and review of evaluation data highlighted several system challenges that should be considered prior to implementing the NP role at other sites. Some included system challenges, staff education on scope of practice, as well as NP clinician turnover. The role must be seen as value added, rather than competition with physicians. The overarching focus should be on appropriate patient care. All staff, including clinicians and non-clinicians, need to be educated about the role including NPs' education, skill set, and competencies. Site leadership support, both verbally and in action is also integral to proper integration of NPs. It should also be ensured that there is a support team/evaluation team to assist with data collection and analysis.

\section{Conclusion}

In conclusion, NPs are in a position to provide support and promote high-quality patient care in the ED setting to ensure patients are managed in an efficient and timely manner. NP-led clinics can also support the ED by ensuring timely follow-up, including medical assessments and changes as needed post ED visit, re-connection/initiation to primary care provider, and connection to specialist care as required. An NP-led IVT clinic allows the NP to be first point of contact into IVT. From triage to initial assessment of all IVT the NP is involved in both independent and collaborative practice providing safe and effective care. Overall, the finding of Tessera \& Gibbons-Reid (2015) indicate that NPs optimize services at $\mathrm{SCH}$, improve access and patient flow, not only at $\mathrm{SCH}$, but throughout the healthcare system in the Edmonton Zone.

\section{Take away points}

1. NPs improve patient flow through the ED by initiating treatments and diagnostics in the ED waiting room.

2. NPs follow up on diagnostics and laboratory results to ensure appropriate treatment was provided and to increase the ability for ED providers to see patients.

3. NPs provide care to patients in the IVC, which helps to decrease hospital admissions and prevent return visits to the ED.

4. NPs provide follow-up for ED patients in the EDT and prevent return visits to the ED.

\section{Acknowledgements}

Alberta Health Services (AHS) managers, physicians, and evaluation team.

\section{About the authors}

\section{Wesley Shand, NP, MN, BScN, ACCN}

Nurse Practitioner at Strathcona Community Hospital in the Emergency Department, IV Clinic and Emergency Transition Clinic. Nurse Practitioner in corrections at the Fort Saskatchewan Correctional Centre(FSCC).

Wesley has been a full time nurse practitioner for 5 years at the Strathcona Community Hospital site in Sherwood Park, Alberta. He has also worked in corrections at the FSCC on a casual basis for 2 years. Prior to his work as a nurse practitioner, he was a registered nurse for 10 years in several intensive care units with the majority of time in the Cardiovascular ICU at the University of Alberta/ Mazankowski Alberta Heart Institute. He enjoys spending time with his family, coaching soccer, reading and camping.

Daris Klemmer, MN, NP-FAA, GNC(c)

Daris has worked as a Nurse Practitioner in a variety of environments, including most recently as the NP Clinical Lead at Strathcona Community Hospital. She holds a Bachelor of Nursing and a Master of Nursing: Advanced Nursing Practice, both from Athabasca University. Daris has diverse Nurse Practitioner and overall nursing experience in both urban and rural settings. She 
has participated extensively in quality and best practice initiatives in the Edmonton Zone: examples include development of an Antimicrobial Stewardship Program at STCH and work with zone partners to promote STCH's innovative care model in the IVT/ Emergency Transition Clinic.
Daris has taught at both the undergraduate and graduate nursing levels, and is currently an instructor at Athabasca University in the Graduate NP Program. She is an active member of the Alberta Health Services, Advanced Practice Nursing community. She brings significant nursing expertise and enthusiasm to the Edmonton Zone Professional Practice Leader role.

\section{References}

Canadian Nurses Association. (2006). Practice Framework for Nurse Practitioners in Canada. Retrieved from https://www. cna-aiic.ca/-/media/cna/page-content/pdf-en/04_practiceframework.pdf $?$ la $=$ en \&hash $=$ CEF 6358183F07D3D3 190 E6AEE6FAE510E06460E0

CARNA. (2017). Scope of Practice for Nurse Practitioners. Retrieved from https://www.nurses.ab.ca/docs/default-source/documentlibrary/standards/scope-of-practice-for-nurse-practitioners. pdf?sfvrsn=ab5ce4b9_10

Claeys, K. L. (2015). Acute bacterial skin and skin structure infections. treated with intravenous antibiotics in the emergency department or observational unit: Experience at the Detroit Medical Center. Infectious Diseases and Therapy, 4(2), 173-186.

El-Jardali, F. \& Lavis, J. N. (2011). Issue brief: Addressing the integration of nurse practitioners in primary healthcare settings in Canada. Hamilton, Canada: McMaster Health Forum; 6 July.

Government of Alberta. (2017). Alberta Health Primary, Community and Indigenous Health - Community Profile: Sherwood Park.

Jennings, N., Clifford, S., Fox, A., \& O'Connell, J. (2015). The impact of nurse practitioner services on cost, quality of care, satifaction and waiting times in the emergency department: A systematic review. International Journal of Nursing Studies, 421-435.

Lutze, M., Fry, M., Mullen, G., O’Connell, J., \& Cotes, D. (2018). Highlighting the invisible work of emergency nurse practitioners. The Journal for Nurse Practitioners, 14(1), 26-32.

Nestler, D., Fratzke, A., Church, C., Scanlon-Hanson, L., Sadosty, A., Halasy, M., ... Hess, E. (2012). Effect of a physician assistant as triage liaison provider on patient throughput in an academic emergency department. Acad Emerg Med, 19(11), 1235-1241.

Olsen, B., Yu, S., \& So, L. (2018). STRATHCONA Community Hospital Integrated Model of Care: Year 4 Evaluation. Alberta Health Services.

Rowe, B., Channan, P., Bullard, M., Blitz, S., Saunders, L., Rosychuk, R., ... Holroyd, B. (2006). Characteristics of patients who leave emergency departments without being seen. Academic Emergency Medicine, 848-852.

Statistics Canada. (2016). Edmonton Census Profile, 2016 Census. Retrieved from https://www12.statcan.gc.ca/ census-recensement/2016/dp-pd/prof/details/page.cfm? Lang $=\mathrm{E} \& \mathrm{G}$ e o $1=\mathrm{CSD} \& \mathrm{Code} 1=4811061 \& \mathrm{Ge}$ o $2=$ CMACA \&Code $2=835 \&$ Data $=$ Count $\&$ SearchText $=$ edmonton $\&$ SearchType $=$ Begins $\&$ SearchPR $=01 \& B 1=$ All $\&$ TABID $=1$

Tessera, E., \& Gibbons-Reid, V. (2015). Integrating nurse practitioners at STRATHCONA Community Hospital evaluation report. Alberta Health Services, Research Priorities \& Implementation, Edmonton.

Tessera, E., \& Gibbons-Reid, V. (2016). Integrating Nurse Pratitioners at STRATHCONA Community Hospital: Year 2 Evaluation Report. Alberta Heath Services.

Tessera, E., \& Gibbons-Reid, V. (2017). Integrating Nurse Practitioners at STRATHCONA Community Hospital: Year 3 Evaluation Report. Edmonton.

Tubman, D. M. (2019). Personal Interview from Jan 7, 2019. 